\title{
Literatur-Verzeichnis
}

\section{Geburtshülfe. Allgemeines.}

Arnstein, E., Zur Prophylaxe in deГ Geburtshülfe. Prager med.

Wochenschr. 25. Juni. S. 341. Brown, E. K., A plea for stricter prophylaxis and more scientific management of obstetrical cases in Tenement House Practice.

Med. Record. 16. Mai. S. 810. Freund, E., Ein Fall von Schwangerschaftmyelitis. Prager med.

Wochenschr. 18. Juni. S. 327. Jung, Ph., Beiträge zur frühesten Ei-Einbettung beim

menschlichen

Weibe. Mit 20 Figuren auf 7 Tafeln. S. Karger, Berlin. Königstein, H., Über das Schicksal deГ Spermatozoen, welche nicht zur

Befruchtung gelangen. (Aus d. phys. Inst. der Univ. Wien.) Wiener

klin. Wochenschr. 2. Juli. S. 971. Kreidl, A. und Mandl, L., Über experimentell erzeugte

Verlängerung der

Tragdauer bei Kaninchen. Wiener klin. Wochenschr. 4. Juni.

S. 823. Longridge, Die Ausscheidung von Creatinin bei Kreissenden und Be-

merkungen zur Rückbildung des Uterus. Journ. of Obst. and Gyn.

of Brit. Emp. Juni. S. 420. Neu, Untersuchung über die Bedeutung des Suprarenins fur die Geburtshülfe. Arch. f. Gyn. 85. Bd. S. 617. Ribemont-Dessaignes, Leçon d'ouverture. Annales de Gynécologie et

d'obstétrique. Mai. S. 257. Schultze, B. S., Über Schwangerschaftsdauer, letzte Regel und Empfäng-

nis. Gyn. Rundsch. 14. Heft. S. 357. Wasenius, Experimentelle Untersuchungen über die

Uteruskontraktionen

bei der Geburt, sowie üh $\beta \Gamma$ den Einfluss des Äthers und des

Morphiums auf dieselben. Arbeiten aus der geb.-gyn. Klinik von

Helsingfors. H. 11.

Physiologie und Pathologie der Schwangerschaft, der Geburt und des Wochenbettes.

Albeck, V. und Lohse, J. E,, Ein Versuch, das Eklampsiegift auf experi-

mentellem Wege nachzuweisen. Zeitschr. f. Geb. u. Gyn. LXII.

S. 115. Arnstein, E., Beitrag zur Porro-Operation. Prager med. Wochenschr.

25. Juni. 1900. S. 743. Barbour, A. H. F., Das untere Uterinsegment. Zeitschr. f. Geb. u. Gyn.

LXII. S. 91. Basso, Delia stafilóccocemia puerperale. La Ginec. 30. April. S. 225. Bighanie und Barabarchi, Sulla provocazione del parto prematuro

250

Literatur - Verzeichnis.

inquanto riguarda i metodi psati. La rassegna d'ostetr. e gin.

Mai. S. 157. Cíulla, M., Studio clinico e medico-legale della gavidanza tardiva. La

Gin. Moderna. Mai. S. 258. Ghampneys, Über einen Fall von plattem Becken und Einleitung der Frühgeburt. Journ. of Obst. and Gyn. of Brit. Emp. S. 430. Clivio, A proposito di un caso di mola vesicolaro inizial $\beta$. Folia 
gynaec. I. S. 138. Guff, Klinisch $\beta$ Berichte über einige Ursachen von Peritonitis während der Schwangerschaft. Brit. med. Journ. Juli. S. 17. Czyzewicz, Adam pin., Hyperemesis gravidarum Samml. klin. Vortr.

N. F. 485 (Gyn. 176). Dannreuther, W. T, Klassischer Kaiserschnitt an einer Zwergin mit gutem Erfolg $\beta$ für Mutter und Kind. Med. Record. 6. Juni. S. 933. Davis, C, The blood pressure in eclampsia. University of Penna.

Med. Bull. XXI. 3. S. 75. Ekstein, 1st die Uterusperforation bei der Abortbehandlung zu vermeiden? Gyn. Rundsch. 11. H. S. 409. Elgood, O., Fall von Pyelonephritis in der Schwangerschaft, geheilt ohne Unterbrechung derselben durch Diät und Urotropin. Brit. med. Journ. S. 1424. Fraenkel, L., Ovarialantikörper und Osteomalacie. Munch, med. Wochenschr. 25. S. 1327. French, H., The Goulstonian lectures on the influence of pregnancyupon certain medical diseases and of certain medical diseases upon pregnancy. Brit med. Journ. Mai. S. 1100. Gallata, E., Supravaginale Amputation eines 16 Wochen graviden Uterus wegen hòchstgradiger Osteomalacie. Gyn. Rundsch. 11. H. S. 405. Gaus, H., Spontane Uterusruptur im Beginne der Geburt. Deutsche med. Wochenschr. No. 28. 9. Juli. S. 1230. Halpenny, Sectio caesarea und über die Indikation zu diesem Eingriffe.

Amer. Journ of Obst. Juni. S. 793. v. Herff, Gemässigter oder radikaler Konservatismus in der Behandlung

des engen Beckens? Munch, med. Wochenschr. 2. Juni. S. 1176. Derselbe, Über die Bewertung gewisser Behandiungsmethoden der

Bakteriämien des Kindbettfiebers, insbesondere der Hysterektomie.

Deutsche med. Wochenschr. No. 26. 25. Juni. S. 1137. Hörrmann, Die Konjunktivalreaktion bei Adnexerkrankungen. Munch.

med. Wochenschr. 26, S. 1375. Kleineríz, Zwei Fälle von Nierßndekapsulation bei Eklampsie. Centralbl.

f. Gyn. N. 26. 21. Juni. S. 843. Koivnatzky, Geburtsstörung durch vaginale Fixation. Munch, med.

Wochenschr. 19. Mai. S. 1078. Kre ¿sch, Sectio Caesarea vaginalis bei Eclampsie. Munch, med. Wochenschr. 2. Juni. S. 1183. Leopold, G., Über akute Peritonitis vor und in der Geburt. Centralbl.

f. Gyn. 11. Juli S. 905. Levi, Contributo allo studio del distacco di placenta normalmente inserta. La rassegna d'ostr. e ginec. April und Juni.

Literatur - Ver zeichnis.

251

Mc Garthy, An address an puerperal eclampsie, with special reference to its treatment with Nitro-Glycerine. Brit. med. Journ. 23. Mai.

S. 1220. Maldé, de, II parto prematuro provoccto nella pneumonite crupale.

La Rassegna d'Ostetr. e Gin. Juni. S. 347. Mathes, P., Wochenbettfieber und Hebammenfrage. Gyn. llundsch.

H. 10. S. 363. Mayer, Aug., Die beckenerweiternden Operationen (mit Vorwort von

A. v. Rosthorn). 1908 S. Karger. Möhlmann, K., Über die Therapie der Ek'ampsie. Zeitschr. f. Geb. u.

Gyn. LXII. S. 79. Müller, S., Drei Fälle von Zentral-Ruptur des Dammes. Gyn. Rundsch. 
H. 10. S. 361. Müller, Zur Serumbehandlung des Puerperalfiebers. Munch, med.

Wochenschr. 19. Mai. S. 1069. Normandíe, R. L. de, Bericht über 250 Geburten in

Hinterhauptslage

aus der Bostoner Gebäranstalt. Surgery. Gyn. and Obst. April.

S. 401. Poïlak, Sammelreferat über Eklampsie. Gyn. Rundsch. H. 10. S. 370. Rosenfeld, Über das Frühaufstehen im Wochenbett. Gyn. Rundsch.

H. 11. S. 401. Runge, E., Appendicitis während der Schwangerschaft, Geburt und

Wochenbett. Berk klin. Wochenschr. 6. Juli. S. 1277. Ruth, W., Zwei Fälle von Hydrorrhoea uteri gravidi amnimalis. Centralbl.

f. Gyn. 11. Juli. S. 913. Sacchi, Per Гeziologia dell'infezione puerperale. Folia gyn. I. S. 53.

Serafini, Ricerche dinamometriche sulla resistenza d $\theta$ lle pariete uterina

alia perforazione coП'istrometro. Rassegna d'Ostetr. e Gin. Mai.

S. 257. Shaw, Chorea in der Schwangerschaft. The Journ. of Obsfc. and Gyn.

of the Brit. Emp. S. 434. Sfameni, Idiorrea cervicale dai canali di Malpighi-Gartner consecutiva

a taglio cesareo demolitore. La Rassegna d'Ostetr. e Gin. März

u. Mai. Solda, Contributo allo studio del parto spontaneo nelle stenosi pelviche.

Folia gyn. I. S. 97. Tantzscher, Ein Fall von extramembranöser Gravidität. Zentralbl. f.

Gyn. S. 883. Trendeienburg, Zur Operation der Embolie der Lungenarterien. Deutsche

med. Wochenschr. 27. S. 1172. Vineberg, H, N., Pyeliti $\beta$ in Schwangerschaft und Puerperium.

The

Amer. Journ. of Obst. Juni. S 669. Welch, Über Thrombophlebitis. Bulletin of the Lying - In

Hospital of

the City New York. S. 71.

Abortus. - Geburtshülfliche Operationen. - Operationen im

Wochenbett.

Eisenstein, Zwei Fälle spontaner Uterusruptur: 1. Uterusruptur bei tief-stehendem Steisse, 2.

wiederholte Uterusruptur. Zentralbl. f. Gyn. 27. S. 879.

252

Literatur-Verzeichnis.

Kaufmann, Zur Extraktion nach Müller. Zentralbl. f. Gyn. S. 877. Müller, Arthur, Zur Frage der Hebosteotomie und der künstlichen Früh-geburt. Munch, med. Wochenschr. No. 24. 16. Juni. S. 1280 .

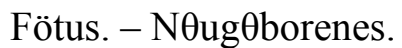

Bondi, Anatomie, Physiologic und Pathologie des Arnnion und der Nabel-

schnur. Sammelreferat. Gyn. Rundsch. H. 11. S. 416. Dewar, T. W., Fall von Blutung aus der

Scheide und Milch-S $\theta$ kretion

bei einem Kinde. The Brit. med. Journ. 13. Juni. S. 1423. Grüneberg, Zur Behandlung der

hämorrhagischen Diathese bei Neu-

geborenen. Munch, med. Wochenschr. 19. Mai. S. 1079. Hengott, A., Considerations sur

Гérysipèle chez le nouveau-né. Annales

do Gyn. et d'Obst. Mai. S. 288. Klose, H., Moderne SäuglingspfJege und Fürsorge. Wiener klin. Rundsch.

23. S. 357. Lambert, S. W., Melaena neonatorum with report of a case cured by transfusion. Med. Record. Mai. S. 885. Leiner, Hautkrankheiten der Neugeborenen mifc Ausschluss der Syphilis. 
Sammelref. Gyn. Rundsch. XII. S. 441. Legge, S. C, Calcium chloride in melaena neonatorum. Brit. med. Journ.

Mai. S. 1173. Moraller, Über Vorbereitungen für diagnostische und tlerapeutische Eingriffe. Gyn Rundsch. H. 11. S. 419. Northcote, A. B., Case of congenital abnormality of hands and feet.

Brit. med. Journ. Mai. S. 1107. See/elder, Über fötale Augenentzündungen. Deutsche med. Wochenschr.

28. S. 1233. Stowe, H. M., Über die Todesursachen von während der Geburt gestorbenen reifen Früchten. Surgery. Gyn. and Obst. April.

S. 356. Vaccari, Sulle affinità di alcuni momenti eziologici della macГОßomia fetale e della gemellazione. Rassegna. April. S. 200. Waiss, Fötale Chondrodystrophie. The Amer. Journ. of Obst. Juni.

S. 828. Weiland, Kochsalz- und Zucker-Infusion beim Säugling. Berl. klin.

Wochenschr. 28. S. 1309.

II. Gynãkologie.

Allgemeines.

Acconci, Ric $\beta \Gamma$ che sul $\Gamma$ intravazione dell' utero umano. Folia gyn. I.

S. 71. Aichel, O., Eine neue Hypothese über Ursachen und Wesen bösartiger

Geschwiilste. Santiago de Chile. Albrecht, H., Zur Kenntnis der akuten Magenlähmung mit sekundärem

Duodenalverschluss. Centralbl. f. Gyn. 11. Juli. S. 902. Barret, C. W., Irrtümliche Ansichten über den intraabdominalen und den

atmosphärischen Druck als Stütze des Uterus und der anderen

abdominalen Organe. Surgery, Gyn. and Obst. April. S. 369.

Literatur - Verzeichnis.

253

Basso, H. L., Ricerche sui complimenti nel sangue di donna sana, $\mathrm{m} \beta \mathrm{n}$ -

struante, gravida puerpera e loro rapporto coi complementi del

sangue fetale. La Gin. 31. März. Bengelsdorf, ÜbeГ die Reaktion des Scheidensek $\Gamma$ ets. Arb $\theta$ iten aus der

geburtshülflich-gynäkologischen Klinik in Helsingfors. H. 8. Bossí, L'odissea di un $\beta$ levatrice e la triste sorte di una partorient $\theta$ a

proposito della causa trattatasi alia corte d'appello di genova il

giorno 8 febbrario 1908. La Gin. moderna. Mai. S. 251. Cohoe, B. A., Der Durchtritt von

Typtmsbazillen durch die Placenta.

The Amer. Journ. of Obst. Juni. S. 789. Crile, G. W., Der heutig日 Stand der Krebsforschung. The Journ. of

the Amer. med. Assoc. Mai. Grandin, Über den therapeutischen Wert der Behandlung in der Rekonvaleszenz nach abdominalen und vaginalen Operationen. The

Amer. Journ. of Obst. Juni. S. 815. Herl'st, R. H., Serum treatment of gonorrhea, with report of cases. The

Journ. of the Amer. med. Assoc. Mai. S. 1678. Hirschberg, Beitrag zur Behandlung mit

Oophorin. Munch, med. Wochen-

schrift. 25. S. 1330. Keller, Zwei Fälle von kongßnitalem Sakraltumor. Arch. f. Gyn. Bd. 85.

S. 555. Math.es, P., Beobachtungen an mit Placentasaft durchströmten Hunde- 
nieren. Zeitsclir. f. Geb. u. Gyn. LXII. S. 108. Meissl, Th., Über den Wert der intravenösen Adrenalin-Kochsalz-In-

fusion. Wiener klin. Wochenschr. 4. Juni. S. 835. Moraller, Über Vorbereitungen für

diagnostische und therapeutische Ein-

griffe. Sammelref. Gyn. Rundsch. XII. S. 443. Paramore, Die Hauptstützpunkte der weiblichen

Beckeneingeweide. The

Journ. of Obst. and Gyn. of the Brit. Emp. S. 391. Rebaudi, Stf., L'Azoto colloidale nell' urine delle donne durante la

gravidanza, il travallo di parto ed il puerperio. La Gin. moderna.

Mai. S. 231. Russ, Über Varicenbildung an den unteren Extremitäten. Surgery.

Gyn. and Obst. April. S. 384. Winckel, Fr. v., Frauenleben und -leiden am Äquator und auf den Polar-

eisen. Samml. klin. Vortr, N. F. 481. Gyn. No. 175. Zanfrogini, L'indirizzo ginecologico n $\beta 11 \mathrm{a}$ scuola e nella pratica. La

Rassegna d'Ost. e Ginec. Juli. S. 321.

Vulva. - Scheide.

Assereto, Sul carcinoma della vulva. Folia Gynec. I. S. 1. Cordaro, Marsupializzazione o sutura dei tumori cistici degli annessi

uterini non radicalmente operabili. La Rassegna d'Ostetr. e Gin.

Mai/Juni. Hinselmann, N., Beitrag zur Kenntnis der bösartigen pigmentierten

Geschwülste der Vulva. Zeitschr. f. Geb. u. Gyn. LXII. S. 34. Müller, Alb., Zwei Fälle von

Scheidenverletzungen sub coitu. Gynäk.

Rundsch. XII. S. 434.

254 Literatu $\Gamma$-Verzeichnis.

Ward, Über das Unterlassen von plastischen Operationen am Becken-

ausgang. The Amer. Journ. of Obst. Juni. S. 821. Weißswange, Vulvakarzinom. Gyn. Rundsch.

10. Heft. S. 361.

Uterus und Operationen an demselben.

Atthill, On the causes of inversion of the uterus. British Med. Journ.

23. Mai. S. 1224. Bland-Sutton, Berielit über den gegenwärtigen Stand der abdominalen

Myomotomie in London. British med. Journ. 4. Juli. S. 1. Davis, Syncytiom oder

Chorioepitheliom der Placenta. Surgery. Gyn.

and Obst. Mai. S. 473. Dührssen, Die Keilresection des Corpus uteri wegen chronischer Metritis. Arch. f. Gyn. Bd. 85. S. 541. Ernst, Beitrag zuy Kenntnis des Adenomyoma uteri. Arch. f. Gyn. Bd. 85. S. 712. FothergiU, Pathologi $\theta$ und operative Behandlung bei Lageveränderungen der weiblichen Beckenorgane. The Journ. of Obst. and Gyn. of the brit. Emp. Juni. S. 410. FL·ris, Un caso interessante di fibroma del segmento inferiore dell utero.

La Rassegna d'Ost. $\beta$ Gin. Juli. S. 329. Hartmann und Lecéno, Adénome diffus du col utérin simulant le cancer.

Ann. de Gyn. et d'Obst. Mai. S. 297. Puech und Massabuau, Tumeur en grappe du cole de Tutérus. Tumeur

à tissus multiples. Ann. de Gyn. et d'Obst. Mai. S. 306. Schauenstein, Histologische

Untersuchungen über atypisclies Plattenepithel

an der Portio und an der Cervix uteri. Arch. f. Gvn. 85. Bd.

S. 576. Vicarelli, Utero fibromatoso gravido oltre et 4 meso les portalo cora 
amputazione sopravaginale, che, dopo Гamputazione ver spontanea-

mente espelbudo gradatamente Гuovo utero. Folia Gynec. I. S. 33. Wald, 0. L. de, Histological changes in the endometrium incidental

to the menstrual cycle. University of Penna Medical Bulletin. XXI.

3. S. 91. Weishaupt, E., Zur Lehre von der Endometritis und der Bedeutung

der Plasmazellen bei pathologischen Gewebsreaktionen. Zeitschr. f.

Geb. und Gyn. LXII. S. 2. Wilmington, B. S., Ausführliche BeschГ $\beta i b u n g$ eines Falles von

Arterio-

sklerose des Uterus. Surgery, Gyn. and Obst. April. S. 352. Zacharias, P., Zwei Fälle von

Tetanus nach gynäkologischen Operationen.

Erwiderung. Munch, med. Wochenschr. 2. Juni. S. 1185.

Tuben. - Ovarien. - Ligamente.

Al†ieri, Contributo allo studio della torsione del peduncalo nella racolte

tubariche. Folia Gynec. I. S. 37. Góth, Lajos, Bedrohliche innere Blutung aus einem Perithelioma ovarii.

Laparotomie. Heilung. Zeitschr. f. Geb. und Gyn. LXII. S. 26. Harbin, W, P., 3 Fälle von glücklich operierten sehr grossen Ovarial-

cysten. Medical Record. 6. Juni. S. 935. Heinßius, Zur Frage der Rezidive der

Pseudomucinkystome. Gyn. Uundsch.

H. 12. S. 429.

Literatur - Verzeichnis.

255

Maresch, R., Über Salpingitis nodosa. S. Karger.

Mitchell, Primäre Ovarial-Schwangerschaft. Surgery, Gyn. and Obst.

Mai. S. 460. Ries, E., Vater-Pacinische Körperchen in der Tube. Zeitschr. f. Geb.

und Gyn. LХП. S. 100. Rinne, Über Differentialdiagnose von Typhlitis und Adnexerkrankung.

Deutsche med. Wochenschr. 27. S. 1174. Sitzenfrey, Über die Verschleppung von Krebskeimen durch die frei $\theta$

Tube. Gyn. Rundsch. H. 11. S. 393.

Bauchhöhle. - Bauchschnit t. - Scheidenbauchschnitt. Allan Doran, Cystic tumor of the suprarenal body sucessfally removed

by operation. Brit. med. Journ. 27. Juni. S. 1558. Leopold, Zur operativen Behandlung der puerperalen Peritonitis und

Thrombophlebitis. Arch. f. Gyn. Bd. 85. S. 483. Lunckenbein, Der vaginale Kaiserschnitt in der Praxis. Munch, med.

Wochenschr. 27. S. 1387.

Ektopische Schwangerschaft. Holden, Zur Diagnosenstellung der Extrauterin-Schwangerschaft.

The

Amer. Journ. of Obst. Juni. S. 809. Límnel, Anatomische Befunde in einem Falle von

Nebenhornßehwanger-

schaft. Arbeiten aus der geburtshülflich-gynäkologischen Klinik von

Helsingfors. H. 11. Nicholson, Ausgetragene Extrauterin-Schwangerschaft. The Amer. Journ.

of Obst. Juni. S. 801. Searle, F. A., A case of ectopic gestation with unusually early symptoms.

Brit. med. Journ. Mai. S. 1162.

Harnorgane. - Fisteln. Currie, O. J., Five cases of vesico-vaginal fistula. Brit. med. Journ.

Mai. S. 1108. Fenwick, Der Wert der doppelseitigen Uterotomie bei vorgeschrittenem 
Blasen- und Prostata-Karzinom. Brit. med. Journ. 4. Juli. S. 23. Teller, R., Über Incontinentia urina $\beta$ bei Spaltbildung der weiblichen

Urethra und ihre operative Behandlung. Zeitschr. f. Geb. u. Gyn.

LXП. S. 1.

Mamma. Lenk, Zur Frage der Stillfähigkeit. Munch, med. Wochenschr. 19. Mai.

S. 1074. Pfaundler und Moro, Über hämolytisches Ferment in der Frauenmilch.

Munch, med. Wochenschr. 19. Mai. S. 1063.

Narkose. - Anti- und Asepsis. Kaísserlin, R., Geburten im künstlichen Dämmerschlaf. St.

Petersb.

med. Wochenschr. 24. Mai. S. 217. Polano, Kaiserschnitt und Lumbalanesthesie. Munch, med.

Wochenschr.

2. Juni. S, 1178. Testor, Entzündung von Ätherdämpfen in der Expirationsluft einer mit

Äther Narkotisierten. Munch, med. Wochenschr. 2. Juni. S. 1185.

256

Literatur- Verzeichnis.

T $\beta$ chnisches und Akiu $\Gamma$ gie.

Bap, J. und Blake, The use of sterile oil to prevent intraperitoneal

adhesions. A clinical experimental study. Surgery, Gyn. and

obst. Juni. S. 667. Hoffmann, Eine Erîeichterung bei der Urinuntersuchvng. Munch.

med. Wochenschr. 25. S. 1338. Hollmann, W., Ein Schwitzapparat mit Ventilationsvorrichtung. St.

Pet $\beta \Gamma$ sb. med. Wochenschr. 17. Mai. S. 205. Knapp, L., Ein Vorschlag zur Erîeichterung der Entfernung von Quell -

stiften aus dem Cervicalkanale. Centralbl. f. Gyn. 26. 27. Juni.

S. 841. Leopold, Über die Behandlung des Karzinoms mittels Fulguration durch

Dr. de Keating-Hart. Centralbl. f. Gyn. No. 27. S. 873. Lessona, F., Intomo al impiego della

soluzioni di almateina nella pratice

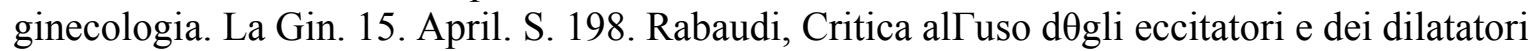

uterini nella

pratica ostetrica, con speciale rigardo all' excitatore Tarnier ei al

dilatatore Bossi. Rassegna. April. S. 203. Thomä, Bemerkungen zu dem Artikel Hammerschlags

„Die Anwendung

der Abortzange”. Centralbl. f. Gyn. No. 26. 27. Juni. S. 846. Wassermann, M., Ein neues

Speculum für Vaginaluntersuchung bei

intaktem Hymen. Deutsche med. Wochenschr. No. 26. 25. Juni.

S. 1146.

Verantwortlicher Redakteur: A. Martin in Berlin. 